\title{
El agua y sus manifestaciones en el léxico de argot: un estudio comparativo ${ }^{1}$
}

\section{Pozas-Ortega, María Nieves}

Universidad de La Laguna, mpozas@ull.es

\section{Resumen}

En esta comunicación abordamos el estudio de algunas denominaciones del agua a través de ejemplos extraídos de la lexicografía argótica, tanto en francés como en español. Una vez analizados los diferentes procedimientos de creación léxica en la parcela que nos ocupa, nos centramos principalmente en el tratamiento histórico de estas voces en la lexicografía especializada así como en el estudio de su vigencia y empleo en la actualidad.

Palabras clave: agua; argot; lexicografía; lexicología.

\section{Résumé}

Dans cette communication, nous abordons l'étude de quelques-unes des dénominations de l'eau à travers des exemples extraits de la lexicographie argotique, aussi bien en français qu'en espagnol. Après avoir examiné les différents procédés de création lexicale mis en ouvre dans le vocabulaire qui nous occupe, nous axons notre analyse sur le traitement historique de ces vocables dans la lexicographie spécialisée ainsi que sur l'étude de leur vitalité et usage aujourd'hui.

Mots-clés: eau; argot; lexicographie; lexicologie.

\begin{abstract}
The aim of this work is to study some of the different names given to water through examples taken from slang dictionaries both in French and Spanish. Once the different mechanisms of lexical creation are analysed in the field under study, the focus is placed mainly on the historical approach to these words in specialised lexicography, and also on the study of their validity and use at present.
\end{abstract}

Keywords: water; slang; lexicography; lexicology.

\footnotetext{
${ }^{1}$ Este trabajo forma parte de una investigación llevada a cabo en el marco de una beca de movilidad cofinanciada al 85\% por el Fondo Social Europeo (Resolución de 29 de octubre de 2008)
} 


\section{Introducción}

Elemento básico en el desarrollo de la vida, el agua es uno de los principales componentes de los organismos vivos, y el más abundante de la superficie terrestre. Siendo, además, el líquido que forma la lluvia, el mar, los ríos, etc., nos preguntamos qué cabida tiene este elemento en el léxico de argot, tanto en francés como en español. Se trata pues de un estudio comparativo que pretende bucear en una parcela léxica algo relegada en España respecto a lo que se ha venido publicando en el país vecino, como se desprende de la ingente bibliografía reseñada únicamente en lo que a lexicografía especializada se refiere.

Como el análisis de todas las unidades acopiadas sobrepasaría los límites de esta comunicación, nos centraremos principalmente en aquellas que aluden al agua como bebida y al agua de lluvia. Así, en el corpus francés, hemos seleccionado una treintena de formas, muchas de las cuales remontan al siglo XIX, época en la que proliferan todo tipo de repertorios lexicográficos. En cuanto al corpus español, más reducido, sólo hemos retenido seis, dos voces para el siglo XVII, dos voces para el siglo XIX, y dos locuciones registradas en el siglo XX.

\section{El agua y sus manifestaciones en el argot antiguo: jargon y germanía}

Aunque estamos utilizando la voz argot, que no se documenta en Francia hasta 1628, es preciso puntualizar que el vocablo más usual para referirse a los primeros períodos de este fenómeno esencialmente oral es jargon, «la plus ancienne expression pour désigner en France l'argot des malfaiteurs» (Dauzat, 1956: 10)². En España, además del equivalente jerga, préstamo del francés, se emplea la voz germanía, con la que se designa históricamente la lengua secreta que usaban entre sí los ladrones, pícaros y rufianes españoles de los siglos XVI y XVII ${ }^{3}$. Conviene recordar, además, que tanto el léxico argótico en Francia como el léxico germanesco en España gozaban de gran difusión en el siglo XVII gracias a la fascinación por la literatura picaresca.

\subsection{Ance, lance}

Corresponden a este periodo las formas ance, lance. Con el significado de «eau», la grafía ance (1562) ${ }^{4}$, que viene recogida por uno de los primeros vocabularios argóticos destacados en la historia del argot francés, La Vie généreuse, de 1596, figura en dos de los diccionarios de nuestra documentación que lo representan tanto diacrónica como diastráticamente mediante una serie de símbolos que hacen referencia al grupo en el que se localiza la voz, «malfaiteurs», a las fuentes utilizadas, o a su pervivencia, señalada ya como caída en desuso en este caso ${ }^{5}$. Más frecuente es la forma lance, documentada igualmente en 1562, y que encontramos en otros textos argóticos capitales del siglo XVII, como el Jargon de l'Argot (1628), aunque se emplee ya en la Quinzième Serée de Guillaume Bouchet (1597): «pier de lance, boire de l'eau» (1608: 97).

En cualquier caso, se trata de una palabra antigua de origen incierto ${ }^{6}$ que comparten los argots románicos. La germanía española recoge la forma ansia con el significado de «agua» y «tormento de agua», tal como aparece en dos entradas del Vocabulario de Hidalgo (1609), junto a una tercera, en plural, para referirse a las galeras. Ansia aparece incluida por

\footnotetext{
${ }^{2}$ Otras denominaciones más o menos antiguas utilizadas en francés para referirse al argot son jobelin, bigorne, blesquien, latin, langue verte, narquois, etc. Cf. Sainéan (1912), Dauzat (1956), TLFi, s.v., etc.

${ }^{3}$ Otros términos usuales para este periodo son jácara, jacarandina, jerigonza, etc., mientras que germanía moderna, caló jergal, caló delincuente, taleguero o talegario, caliente, argot, etc., aluden al argot moderno, cf., entre otros, Clavería (1941; 1967), Daniel (1991), Sanmartín (1998; 2006). Para un panorama completo de la aparición del vocablo argot, préstamo del francés, en la lexicografía española, véanse los trabajos de Buzek (2011a; 2011b).

${ }^{4}$ La voz se encuentra ya en jobelin o «argot de la fin du XVe siècle»: «[Servis] d'ance plaisant, d'eau joyeuse, c.-à-d. de vin»(Esnault, 1965, s.v. ance).

${ }^{5}$ Cf. Delesalle (1896): «ance, anse • (B) n.f. Eau». Sobre esta inicial, no especificada en las «Abréviations», el autor parece referirse a Blesquien, término que aparece en el glosario que acompaña a la Vie généreuse (1596). En cuanto al símbolo •, se alude al «argot des malfaiteurs». En adelante prescindiremos de la simbología. Cf. Bruant (1901) ance*, anse*, s.v. eau, en las que el $(*)$ acompaña «les mots anciens, démodés ou tombés en désuétude dont on ne sert plus ou presque plus».

${ }^{6}$ Cf. «Fourb. lenza, eau (XVe s.); le germ. ansia, id. a subi la même aphérèse du l. - V. lance» (Esnault, 1965, s.v. ance); «Arg. italien lenza et, davantage, slenza, fleuve, pluie, urine, boisson, subst. verbal de slenzare, uriner (XVe s.), issu du bas lat. lanceare, comme le franç. lancer [...]. // Var. ance (le $l$ initial ayant été pris pour un article), v. ce mot» (Esnault, 1965, s.v. lance). Cf. TLFi (s.v. lance) y DFNC (1991, s.v. lance), entre otros.
} 
Salillas en su «Vocabulario de germanía» (1896); también la tuvo en cuenta Besses (1905), acompañándola de la marca correspondiente a «argot gitano o caló» ${ }^{8}$.

Otro vocablo de germanía registrado por Hidalgo (1609) y catalogado como tal por Salillas (1896) es clariosa «agua». El diccionario de Besses (1905) distingue clarisa «agua» como voz de caló y clariosa «ventana» como argot delincuente, única acepción que traen algunos repertorios lexicográficos contemporáneos, como Ruiz (2001) ${ }^{9}$. El diccionario de la Academia en su vigésima tercera edición (2014) anota clariosa como voz de germanía, definiéndola como «agua natural» y explicándola a partir de clara. En la acepción general de «agua», clariosa es sustituida frecuentemente por ansia, de igual significado, aunque en algunos romances de germanía también se emplea para evocar las lágrimas (Alonso, 1979; Chamorro, 2002).

\section{La expresión del agua en el argot moderno}

El siglo XIX se caracteriza por la irrupción en el mercado lexicográfico francés de una cohorte de repertorios de argot, de jargon parisien, d'excentricités du langage parisien, de langue verte, etc. La noción de argot ya no hace referencia al lenguaje de los bajos fondos exclusivamente sino que comprende los lenguajes especiales profesionales y la lengua popular, aunque algunos autores sigan prefiriendo la denominación de argot para el vocabulario de la delincuencia y de la marginalidad (l'argot du milieu), y centren sus investigaciones en este vocabulario ${ }^{10}$. Ahora bien, en este contexto, no es raro encontrar tentativas de delimitación de las voces objeto de estudio en función de los grupos de usuarios, por lo que se añaden anotaciones como «dans l'argot des malfaiteurs», «dans l'argot des chiffoniers», «dans l'argot des faubouriens», «dans le jargon des ivrognes», «dans l’argot du peuple», «dans le langage familier», entre otras.

\subsection{Del ratafia(t) de grenouille(s) al sirop de barbillon}

El vaciado de los repertorios de argot arroja un gran número de expresiones que incluyen entre sus componentes un nombre de líquido, de consistencia y de naturaleza diversa, generalmente un licor (ratafía, anisette, élixir, etc.) y un nombre de animal acuático, como peces de agua dulce (ablette, barbillon, goujon), aves (canard), y batracios (grenouille, crapaud). Pero no es raro encontrar referencias a objetos de la vida cotidiana, siempre y cuando estén relacionados con el agua, como ocurre con el paraguas (parapluie, pébroque). Veamos algunas de estas locuciones que no hacen más que desarrollar un modelo antiguo de denominación jocosa del agua, como se desprende de la locución vin de grenouilles que evoca Larchey (1889).

Entre las expresiones que utilizan un nombre de licor y de un animal acuático, como las ranas, cuyo hábitat natural son las zonas húmedas, conviene mencionar, en primer lugar, la locución ratafía(t) de grenouille(s), que el TLFi (s.v. ratafia) califica de «pop. vieilli», documentándola desde $18288^{11}$. Los diccionarios de argot recogen esta expresión con el significado de «eau» y de «pluie» (Nain, 1847, ratafiat de grenouille) ${ }^{12}$. En estos ejemplos se parte del nombre de un aguardiente a base de azúcar y fruta utilizado por sus propiedades estomacales, y de un batracio asociado tanto a la lluvia como al agua de mala calidad.

\footnotetext{
${ }^{7}$ «Del lat. anxius, angustiado, afligido. f. Agua.|| Tormento de agua. || pl. Galeras. || La representación es de origen esencialmente procesal. Deriva del tormento de toca (V. pág. 55). Por eso, y después por simpatía hacia el vino, se llama ansia el agua. La pena de galera, por cumplirse navegando, equivale a un segundo tormento de agua» (Salillas, 1896: 268-269). Cantar en el ansia se recoge en el DRAE-2014 con la marca de germanía: «confesar en el tormento, especialmente en el de toca», del latín tardío anxia, de la raíz de angěre 'estrechar','ahogar'. Sobre la presencia de esta y otras voces germanescas en jergas no delincuentes o gremiales, cf. Salillas (1896: 251-252) y García González (1975: 399), entre otros.

${ }^{8}$ Besses incluye voces del caló (c.) extraídas del vocabulario de Salillas y de fuentes lexicográficas gitanas no especificadas; también marca como argot delincuente (d.) voces señaladas como de germanía en Salillas (Sanmartín, 2004: 726; Buzeck, 2011a: 62). Otras, como ansio «río», llevan una marca «prov.» (¿provincialismo?) que no figura en el cuadro de «Abreviaturas».

${ }^{9}$ Cf. el tratamiento de este vocablo en el repertorio gitano de Pabanó (1915): *clariosa s. agua // ventana, donde el (*) indica que se trata de una voz germanesca, como se precisa en la nota 1 (s.v. abacería).

${ }^{10}$ Aspecto abordado por Sainéan (1912), Dauzat (1956), Guiraud (1956), Clavería (1941), Daniel (1991), Sanmartín (1998; 2006), entre otros.

11 «Il te fallait [étant jardinier] empoigner l'arrosoir, et te morfondre à jeter du ratafia de grenouilles sur tes tulipes» (Vidocq, Mémoires, t. 2, 1828-29, p. 47). El TLFi cita, también, un ejemplo de liqueur de grenouilles en 1875.

${ }^{12}$ En algunos repertorios se precisa el origen social de la expresión, a modo de indicación diastrática: «dans l’argot du peuple» (Delvau, 1866², ratafiat de grenouille), o «dans le jargon des ivrognes» (Rigaud, 1888, ratafia de grenouilles).
} 
Las siguientes entradas parten de nombres de peces de agua dulce, como el barbillon y el goujon: Anisette de barbillon $(1864)^{13}$ viene recogida ya por Delvau (1866) como «argot du peuple» ${ }^{14}$. Algunos autores la identifican como «Expression usitée dans les prisons [...]», explicándola por «Allusion au poisson de ce nom qui vit dans l'eau claire» y etiquetándola como «argot des voleurs» (Virmaître, 1899). Como puede observarse, se trata de un esquema de formación muy productivo en la designación metafórica del agua que ha generado otro tipo de expresiones sinónimas. Sirvan de ejemplo kirsch de barbillon, documentada desde 1870 (Esnault, 1965, «pop.»), ratafia de barbillon (Bruant) y anisette de goujon (Rossignol), locuciones estas últimas menos usuales que traen estos repertorios de 1901.

Como expresión sinónima de la locución anisette de barbillon, el TLFi, que señala este empleo figurado como «arg.», cita también élixir de grenouille, en la que aparece nuevamente el tema del batracio. Según las fuentes consultadas, la expresión, frecuente entre 1917 y 1918 en el argot militar, ya es utilizada por Hector France a finales del siglo XIX, quien la recoge también en su diccionario (1907), como sinónima de la locución anisette de barbillon ${ }^{15}$. Menos frecuentes son liqueur des canards, que se encuentra en los textos desde 1842, y que trae Giraud (1989), y ratafia de canard (1901), con nombre de ave acuática en estos ejemplos.

En este tipo de construcciones no es raro el empleo de otras denominaciones de líquidos, como sirop, bouillon, jus, tisane. Veamos algunas de estas locuciones. La imagen del sirop, líquido de consistencia más o menos espesa, aparece en sirop de canard (1845), seguido de la denominación popular del paseriforme acuático. En las siguientes expresiones, menos extendidas, se asocia de nuevo a peces de agua dulce: sirop d'ablette (1863) ${ }^{16}$ y sirop de barbillon (Bruant, 1901, s.v. eau). El motivo del batracio, uno de los más productivos, aparece en sirop de grenouilles (1877) y jus de grenouille (1884), que se acompañan de las marcas «pop. vieilli» en el TLFi (s.v. grenouille). Otras locuciones son bouillon de canard (1838) ${ }^{17}$, con referencia al ave palmípeda, y que también se aplica a la lluvia torrencial desde 1808; en cuanto a bouillon de grenouille, señalada como de «langage familier» (Delesalle, 1896, s.v. grenouille) y tisane de grenouilles (1902), se retoma el tema del batracio esta vez combinado con líquidos de propiedades y de consistencia diversa.

\subsection{De l'essence de parapluie al sirop de pébroque}

Con estas locuciones se alude tanto al agua natural como al agua de lluvia. Con el significado de «eau» y caracterizada como voz popular («dans le jargon du peuple»), la locución essence de parapluie viene en Rigaud (1888), aunque otros diccionarios la recogen con el significado de «pluie», como Delesalle (1896). Otras locuciones sinónimas, aunque menos representadas, son sirop de parapluie (Lermina-Lévêque, 1900) ${ }^{18}$ y jus de parapluie «eau» que se documenta en 1984 (Bob, 2016). Como equivalente de sirop de parapluie, la documentación contemporánea ofrece la locución sirop de pébroque (1960) en la que interviene la voz de argot pébroque «parapluie» (1907), derivada de pépin (1841), voz argótica de igual significado (ColMév, 2010).

Este tipo de formaciones no es exclusivo del siglo XIX puesto que una de las expresiones más antiguas para designar metafóricamente el agua es sirop de l'aiguière, a partir del recipiente de ese nombre. Se trata de una locución jocosa

\footnotetext{
13 «Gautruche, dit Gogo-la-Gaieté, quoi! Un joli garçon à la coule [...] un bon zig qui se la passe douce, et qui ne se donnera pas de colique avec cette anisette de barbillonlà ...» (E. et J. de Goncourt, Germinie Lacerteux, 1864, p. 202, en TLFi, s.v. anisette, «au. fig., spéc., arg.»).

${ }^{14}$ Cf. «On dit aussi anisette de barbillon et Bourgogne de cheval» (Delvau, 1866, s.v. ratafiat de grenouille). Esta última expresión no viene recogida por ningún otro diccionario de nuestra documentación.

15 «-Nom de Dieu ! On voit qu'ils ont pompé et pas de l'élixir de grenouille!» (Les Mystères du monde, 1897-1899: 432). También hay un ejemplo de la expresión sirop de grenouille (p. 124).

${ }^{16}$ «Je songe aux amis qui vont rester dans la chambrée avec lesirop d'ablette[eau] pour consolation». (A. Camus,Les Bohèmes du drapeau,1863, p. 112) (TLFi, s.v. ablette, ilustrando «Quelques emplois fam. de couleur argot. (non signalés dans les dict.)».

${ }^{17}$ Según Larchey (1865). Algunos diccionarios la documentan en Delvau (1866), como ColMév (2010). De la documentación que ofrece Bob (2016) se desprende igualmente el empleo metafórico de canard (1911) para designar al «buveur d'eau». Otra variante, menos frecuente es bouillon de chien, recogida con la acepción de «pluie» (Nain, 1847) y «eau» (Lermina-Lévêque, 1900; Bruant, 1901). Cf. también liquide des canards (Virmaître, 1894, «argot du peuple») y boisson des canards (Giraud, 1989), con un ejemplo de 1928.

${ }^{18}$ «Le buveur, quand il réclame à boire, ne veut pas de sirop de parapluie: seule l’eau bénite de cave peut désaltérer le gosier sec» (Le Monde dimanche, 14 nov. 1982 , p. I) (TLFi).
} 
que se documenta ya en 1649 (TLFi, s.v. sirop), y que recogen algunos diccionarios (Delesalle, 1896, «pop.»; France, 1907).

En cuanto a la locución sirop de baromètre $(1870)^{19}$ se presenta con la siguiente definición: «Eau. Variante sirop de grenouilles» (Rigaud, 1878), aunque también se documenta con el significado de «pluie», probablemente por alusión a la práctica consistente en utilizar a este batracio para anunciar la lluvia, es decir como animal baromètre.

\subsection{De la flotte al château la Pompe}

Además de la forma lance, calificada como «argótica» y «popular» («arg., pop.») en el TLFi, y que prevalece en la actualidad en todas las acepciones que ha desarrollado esta voz a lo largo del siglo XIX ${ }^{20}$, conviene mencionar flotte, de origen incierto. Las acepciones documentadas de «rivière, mer, pluie, boisson», «populares» en 1886-1895 (Esnault, 1965), son consideradas hoy simplemente «familiares», como coinciden en señalar la mayoría de autores (TLFi; PRob, 2008; DFNC, 1991; ColMév, 2010) ${ }^{21}$.

En la designación del agua potable (del grifo), por alusión a los grandes caldos franceses, como los Château-Lafitte, la documentación ofrece desde finales del siglo XIX la expresión irónica y jocosa cru du château la Pompe (Virmaître, 1894, «par ironie», «argot du peuple»), abreviada generalmente en Château-La-Pompe (Lermina-Lévêque, 1900, s.v. eau). La trae el TLFi como «pop. et p. plaisant.» (con la grafía Château-la-pompe), mientras que el PRob (2008) sólo la etiqueta como «plais.» (cf. Château-la-Pompe) ${ }^{22}$. El argot español ofrece una expresión similar aunque no recogida por los diccionarios de nuestro corpus: fino cañerías. Usual en el habla castiza de Madrid, se localiza en artículos de periódicos y en blogs extraídos de Internet, con el significado de «vaso de agua» y «agua en general» ${ }^{23}$.

En el corpus español destaca, además, la presencia de dos vocablos tomados del caló, la lengua de los gitanos españoles. Nos referimos a pañí y a jurba.

Pañí «agua», del caló pañí, de igual significado, es una voz registrada por la mayoría de repertorios de argot desde 1905 (Besses) ${ }^{24}$. Sanmartín (2006) la acompaña de la marca «marginalidad» y de una cita de 1999, prueba de su vigencia, frecuente en las coplas andaluzas y en los diccionarios generales de la lengua desde 1853. El tratamiento de este vocablo en el DRAE refleja algunas de las incoherencias de las que adolecen este tipo de obras. Baste señalar que la vigésimotercera edición del diccionario académico (2014) la sigue marcando como voz de germanía, atribuyéndole origen caló ${ }^{25}$, mientras que el DEA (2011) la califica como «jergal».

En el caso de jurba «agua», se trata de un vocablo documentado desde 1866 en el argot catalán, según Salillas (1896: 325) y que recogen algunos diccionarios de argot, como Besses (1905), con identificaciones de «caló» y de «prov.», esta última marca no especificada en las «Abreviaturas»; también lo registra Ramoncín (1993)²6.

\footnotetext{
${ }^{19}$ «Je ne suis pas au sirop de baromètre, entendez-vous bien, vieille bride, de l'eau, c'est bon pour éteindre le feu» (D. Poulot, Le sublime ou le travailleur comme il est en 1870, et ce qu'il peut être, 1980 [1870]) (Bob, 2016).

${ }^{20}$ Cf. «rivière» (1835), «pluie» (1836), empleo más frecuente, aunque también puede nombrar cualquier otro líquido corporal, como las lágrimas o la orina desde 1836. Los derivados lanscailler (1628), lancecailler (1957) «uriner», con el suf. arg. - caill, lansquiner, lancequiner (1800) «pleuvoir», han servido a su vez para formar los sustantivos lansquine (1866) «pluie», lancequine (1878) «eau», y lanscaille (1957) «eau, liquide, pluie» (ColMév, 2010).

${ }^{21}$ Se documenta primero en el sentido de «bain» (1883), considerado fuera de uso en la actualidad (ColMév, 2010). Otras voces de este periodo son pousse-moulin (Nain, 1847), calificada como "p. plaisant.» por el TLFi, y wallace, utilizada para referirse al agua de las fuentes públicas del mismo nombre, creadas e instaladas por el filántropo británico Sir Richard Wallace en 1872. Esta acepción se documenta desde 1876 en autores de la época (Richepin, Métenier) y en algunos diccionarios de argot (Delesalle, 1896, «pop.»). Dio lugar al verbo wallacer «boire de l'eau» (Rigaud, 1888, «jargon des voyous»).

${ }^{22}$ Para otras «périphrases burlesques», cf. Bob (2016).

${ }^{23}$ Cf. entre otros, el artículo de Moncho Alpuente (2010).

${ }^{24}$ Cf. en locución, caer pañí «llover», entre otras. El corpus francés revela la forma pagnin n.m.«eau» (1954), «mot gitan», según el único repertorio lexicográfico que lo menciona (ColMév, 2010).

25 «Del caló pañí, este del hindi pānī, y este del sánscr. pānīya ‘potable’, 'agua potable’».

${ }^{26}$ Cf. el estudio de este vocablo en Vinyoles i Vidal (1978: 106). Ha desarrollado el compuesto parajurbas «paraguas» documentado en el argot catalán desde 1886 y que traen algunos repertorios tanto de argot (Besses, 1905; Ramoncín, 1993), como de caló (Pabanó, 1915), entre otros. El mismo fenómeno se observa en francés en parelance (pop., 1928) y paraflotte (pop., 1910, 1953; rare) «parapluie» (Esnault, 1965, s.v. lance, flotte)
} 


\section{El agua en el argot contemporáneo: de l'eau qui pique a l'eau à ressort}

El siglo XX aporta algunas creaciones relacionadas con la oposición agua sin gas / agua con gas. Así, frente a l'eau plate, observamos algunas denominaciones como eau qui pique «eau gazeuse» ${ }^{27} \mathrm{y}$, en particular, las referidas al agua de Seltz: eau à ressort (1929) y eau à pédale (1957), «image pittoresque, le gaz fait sauter l’eau» (ColMév, 2010). En el corpus español encontramos la misma imagen en la locución pañí de muelle «sifón», a partir de la voz pañí «agua» ya mencionada. Aunque escasamente representada en la lexicografía argótica (Oliver, 1987; Ruiz, 2001), esta locución referida tanto al sifón como al agua con gas, se registra en la literatura desde 1962 (Ruiz, 2001), y es calificada como «jergal» en el DEA (2011), que también la trae.

\section{Conclusiones}

El léxico analizado constituye un campo semántico bastante «pobre» frente a la abundancia de términos acuñados para nombrar las bebidas alcohólicas o la embriaguez, por ejemplo.

En el corpus francés destacan las locuciones metafóricas, irónicas y jocosas, formadas sobre un patrón muy productivo en ese idioma: la serie sustantivo que designa un líquido (essence, jus, sirop de...) y un animal u objeto relacionado con el agua.

Por lo que toca al argot español, aunque no ofrece un léxico tan abundante como el que se recoge para el francés, sí presenta algunos de los procedimientos usuales en este tipo de lenguas especiales, como es el uso de préstamos, y en particular los préstamos del caló (pañí, jurba), de frecuencia más reducida en la actualidad.

En cuanto a la vigencia del léxico objeto de estudio, hemos visto que las voces que lo conforman pueden considerarse obsoletas en su mayoría. En el caso del francés, constatamos una evolución en las marcas de uso, así por ejemplo flotte, se acompaña hoy de la indicación «familiar». En este sentido, en el corpus español llama la atención la marca de uso «germanía» que sigue utilizando el DRAE para indicar la antigüedad de la voz (cantar en el ansia), o erróneamente, en el caso de algunas voces del caló (pañi) que se mantienen en el habla coloquial.

El recurso de Internet, a través de blogs y foros de naturaleza diversa, permite comprobar la permanencia en el uso de muchas de las voces objeto de estudio, convirtiéndose así en otra herramienta de la que dispone la lexicografía moderna.

\section{Referencias bibliográficas}

AlONSO HERNÁNDEZ, José Luis (1977). Léxico del marginalismo del siglo de Oro. Salamanca: Secretariado de Publicaciones e Intercambio científico. Universidad de Salamanca.

AlPUENTE, Moncho (2010). «Agua va...».<http://elpais.com/diario/2010/05/05/madrid/1273058662_850215.html> [Consulta: 1 de marzo 2016].

BESSES, Luis (1905). Diccionario de argot español o lenguaje jergal gitano, delincuente profesional y popular. Barcelona: Sucesores de Manuel Soler; [edición facsimilar], 1989, Cádiz: Universidad de Cádiz.

Вов (2016). L'autre trésor de la langue, Dictionnaire d'argot, de français familier et de français populaire. <http://www.languefrancaise.net/Bob> [Consulta: 10 de marzo 2016].

Bouchet, Guillaume (1597; 1608). Second Livre des Serees de Guillaume Bouchet, sieur de Brocourt. Paris: Perier.

BRuANT, Aristide (1901). L'argot au XXe siècle. Dictionnaire argot-français. Chez l'auteur; [reproduction], 1990, Chimères.

BuzeK, Ivo (2011a). «La noción de argot en la tradición lingüística hispánica y su primer diccionario: el Diccionario del argot español de Luis Besses (1905)», en Philologia, nº 21, Supplementum II, p. 57-66.

BuZEK, Ivo (2011b). «Argot: Historia documentada de un término en la lingüística española», en Moenia, nº 17, p. $289-302$.

Camus, Antoine (1863). Les Bohêmes du drapeau. Paris: Brunet.

\footnotetext{
${ }^{27}$ Recogida por Giraud (1989), que cita un ejemplo de 1977, y por Bob (2016). Esta expresión se acompaña de la marca «fam.» en el PRob (2008), al igual que la variante eau piquante recogida únicamente por el TLFi desde 1922.
} 
Chamorro, María Inés (2002). Tesoro de villanos: lengua de jacarandina: rufos, mandiles, galloferos, viltrotonas, zurrapas, carcaveras, murcios, floraineros y otras gentes de la carda. Barcelona: Herder.

CHEREAU, Olivier (1628). Le Jargon ou langage de l'Argot Réformé comme il est à présent en usage parmy les bons pauvres, tiré et recueilly des plus fameux argotiers par un pilier de boutanche qui maquille en mollanche en la vergne de Tours, Revu, corrigé et augmenté de nouveau par l’Auteur. Paris: Veuve de Carroy.

Clavería, Carlos (1941). «Sobre el estudio del argot y del lenguaje popular», en Revista Nacional de Educación, n 1, 12, p. 65-80.

ClAVERíA, Carlos (1967). «El argot», en Enciclopedia lingüística hispánica, nº 11. Madrid: CSIC, p. 349-363.

Colmev = Colin, Jean-Paul, Mevel, Jean-Pierre y LeClere, Christian (2010). Le dictionnaire de l'argot et du français populaire. Paris: Larousse. [Nueva edición del Dictionnaire de l'argot. Paris: Larousse, 1990].

DANIEL, Pilar (1991). «Panorámica del argot español», en Victor León, Diccionario de argot español y lenguaje popular. Madrid: Alianza, p. 7-27.

DauZAT, Albert (1956). Les argots. Caractères. Évolution. Influence. Paris: Delagrave.

DeA = SEco, Manuel, AndrÉs, Olimpia y Ramos, Gabino (2011). Diccionario del Español Actual. Madrid: Aguilar. 2ª edición.

DELESALLE, Georges (1896). Dictionnaire argot-français et français argot. Paris: Ollendorf.

DeLVAu, Alfred (1866). Dictionnaire de la langue verte. Argots parisiens comparés. Paris: Dentu.

Delvau, Alfred (21866). Dictionnaire de la langue verte. Argots parisiens comparés. $2^{\mathrm{e}}$ édition entièrement refondue et considérablement augmentée. Paris: Dentu.

DFNC = CELLARD, Jacques y REY, Alain (1991). Dictionnaire du français non conventionnel. Paris: Hachette [1 $1^{\text {a }}$ edición, 1980].

ESNAULT, Gaston (1965). Dictionnaire historique des argots français. Paris: Larousse.

FRANCE, Hector (1897-1899). Les Mystères du monde. Paris: Lachatre.

France, Hector (1907). Dictionnaire de la langue verte. Archaïsmes. Néologismes. Locutions étrangères. Patois. Paris: Librairie du Progrès.

GARCíA GonZÁLEz, Francisco (1975). «El mansolea: una jerga gremial del Oriente de Asturias», en Archivum, n 25, vol. 25, p. 377420.

GIRAUD, Robert (1989). L’Argot du bistrot. Paris: Marval.

GonCOURT, Edmond y Jules de (1864), Germinie Lacerteux. Paris: Charpentier.

Guiraud, Pierre (1956). L’Argot. Paris: Presses Universitaires de France.

HidAlgo, Juan (1609). «Bocabulario de germanía», en Mayans y Siscar, Orígenes de la lengua española. Madrid: Bailly-Ballière, p. 226-267 [edición facsimilar de la de 1737], reimpresión, 1873].

LARCHEY, Lorédan (1865). Les Excentricités du langage. 5 e édition toute nouvelle. Paris: Dentu.

LARCHEY, Lorédan (1889). Nouveau Supplément du Dictionnaire d'argot, avec le vocabulaire des Chauffeurs de l'an VIII et le répertoire de largonji. Paris: Dentu.

LEÓN, Víctor (1991). Diccionario de argot español y lenguaje popular. Madrid: Alianza. [1ª edición, 1980].

LERMinA, Jules y LEVEQUE, Henri (1900). Dictionnaire thématique français-argot, suivi d'un index argot-français à l'usage des gens qui veulent parler correctement la Langue Verte. Chacornac; [reproduction], 1991, Les Éditions de Paris.

NAIN [=Anonyme] (1847). Dictionnaire d'argot ou la langue des voleurs dévoilée contenant les moyens de se mettre en garde contre les ruses des filous, nouvelle édition: Paris.

Oliver, Juan Manuel (1987). Diccionario de argot. Madrid: Sena, $2^{\mathrm{a}}$ ed. aumentada.

Pabanó, F. M. (1915). Historia y costumbres de los gitanos, Colección de cuentos viejos y nuevos, dichos y timos graciosos, maldiciones y refranes netamente gitanos. Diccionario español-gitano-germanesco. Dialecto de los gitanos. Barcelona: Montaner y Simón.

PECHON DE RUBY, pseud. (1596). La Vie généreuse des Mercelots, Gueux et Boesmiens, contenans leur façon de vivre, subtilitez et jargon, Mis en lumière par Monsieur Pechon de Ruby, gentilhomme breton, ayant été avec eux en ses jeunes ans où il a exercé ce beau métier, Plus a été ajouté un Dictionnaire en langage blesquien, avec l'explication en vulgaire. Lyon: Julliéron, avec permission.

Poulot, Denis (1870). Le Sublime, ou le travailleur comme il est en 1870 et ce qu’il peut être. 1980, 2éd. Paris: Maspéro. 
ProB = Le Nouveau Petit Robert (2008). Dictionnaire alphabétique et analogique de la langue française. Paris: Le Robert.

Ramoncín [Martínez Márquez, José Ramón Julio] (1993). El tocho cheli, Diccionario de jergas, germanías y jerigonzas. Ediciones Temas de hoy.

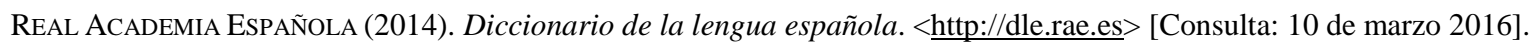

Rigaud, Lucien (1878). Dictionnaire du jargon parisien. Paris: Ollendorf.

RigAuD, Lucien (1888). Dictionnaire d'argot moderne. Nouvelle édition avec Supplément. Paris: Ollendorf.

Rossignol (1901). Dictionnaire d'argot, argot français et français argot. Paris: Ollendorf.

RuIz, Ciriaco (2001). Diccionario ejemplificado de argot. Barcelona: Península.

SAINEAN, Lazare (1912). Les Sources de l'argot ancien. Paris: Champion; 1973, Genève: Slatkine Reprints.

SAlillas, Rafael (1896). El delincuente español. El lenguaje (Estudio filológico, psicológico y sociológico) con dos vocabularios jergales. Madrid: Librería de Victoriano Suárez.

SANMARTín SÁEz, Julia (1998). «Lenguaje y cultura marginal. El argot de la delincuencia» en Cuadernos de Filología, anejo XXV. Valencia: Universitat de València.

SANMARTín SÁEz, Julia (2004). «Los diccionarios de argot español: sus escollos y resoluciones», in Battaner M.P, DeCesaris Janet Ann, De Lexicografía: actes del I Symposium internacional de lexicografía, (Barcelona, 16-18 de maig de 2004), Universitat Pompeu Fabra, IULA, p. 723-740.

SAnMARTín SÁez, Julia (2006). Diccionario de argot. Madrid: Espasa Calpe. [1ª edición, 1998].

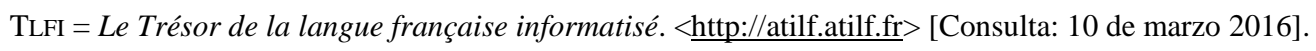

VidocQ, François (1828-1829). Mémoires de Vidocq, chef de la police de sûreté jusqu'en 1827. Paris: Tenon, 4 vol.

VINYOLES I VIDAL, Joan (1978). Vocabulari de l'argot de la delinqüència. Barcelona: Millà.

VIRMAITRE, Charles (1894). Dictionnaire d'Argot fin-de-siècle. Paris: A. Charles.

VIRMAITRE, Charles (1899). Supplément au Dictionnaire d'Argot fin-de-siècle. Paris: A. Charles. 